\title{
De novo missense variants in RAC3 cause a novel neurodevelopmental syndrome
}

\author{
Gregory Costain, MD, PhD ${ }^{1}$, Bert Callewaert, MD, $\mathrm{PhD}^{2}$, Heinz Gabriel, $\mathrm{MD}^{3}$, \\ Tiong Y. Tan, MBBS, PhD ${ }^{4}$, Susan Walker, $\mathrm{PhD}^{5,13}$, John Christodoulou, MBBS, PhD ${ }^{4,6}$, \\ Tamas Lazar, $\mathrm{MSc}^{7}$, Björn Menten, $\mathrm{PhD}^{2}$, Julia Orkin, MD, MSc ${ }^{8,9,10}$, Simon Sadedin, PhD ${ }^{4}$, \\ Meaghan Snell, MS ${ }^{1,11}$, Arnaud Vanlander, $\mathrm{MD}^{12}$, Sarah Vergult, PhD', Susan M. White, MBBS ${ }^{4}$, \\ Stephen W. Scherer, PhD ${ }^{5,13,14}$, Robin Z. Hayeems, PhD ${ }^{10,11}$, Susan Blaser, MD ${ }^{15}$, \\ Shoshana J. Wodak, PhD ${ }^{7}$, David Chitayat, MD ${ }^{1,16}$, Christian R. Marshall, PhD ${ }^{5,11,17,18}$ and \\ M. Stephen Meyn, MD, PhD ${ }^{1,8,11,14,19}$
}

Purpose: $R A C 3$ is an underexamined member of the Rho GTPase gene family that is expressed in the developing brain and linked to key cellular functions. De novo missense variants in the homolog $R A C 1$ were recently associated with developmental disorders. In the RAC subfamily, transforming missense changes at certain shared residues have been observed in human cancers and previously characterized in experimental studies. The purpose of this study was to determine whether constitutional dysregulation of $R A C 3$ is associated with human disease.

Methods: We discovered a RAC3 variant in the index case using genome sequencing, and searched for additional variants using international data-sharing initiatives. Functional effects of the variants were assessed using a multifaceted approach generalizable to most clinical laboratory settings.

Results: We rapidly identified five individuals with de novo monoallelic missense variants in RAC3, including one recurrent change. Every participant had severe intellectual disability and brain malformations. In silico protein modeling, and prior in vivo and in situ experiments, supported a transforming effect for each of the three different $R A C 3$ variants. All variants were observed in databases of somatic variation in cancer.

Conclusions: Missense variants in RAC3 cause a novel brain disorder, likely through a mechanism of constitutive protein activation.

Genetics in Medicine (2019) 21:1021-1026; https://doi.org/10.1038/s41436018-0323-y

Keywords: exome; genome; neurodevelopment; Rho signaling; GTPase

\section{INTRODUCTION}

Rho GTPase signaling is fundamental to cell migration in the central nervous system. ${ }^{1}$ Members of the Rho family of small GTP-binding proteins act as molecular switches that alternate between GTP-bound (active) and GDP-bound (inactive) states via conformational changes in the conserved Switch I and Switch II regions. ${ }^{2}$ The gene RAC3 (MIM 602050) encodes Ras-related C3 botulinum toxin substrate 3, a less studied member of the RAC subfamily of Rho GTPases. RAC3 is expressed in the developing brain and its protein product plays a role in key developmental processes. ${ }^{3}$ However, the phenotypic consequences of constitutional genetic variation in RAC3 are unknown. RAC1 and RAC2 encode related proteins that share $\sim 93 \%$ and $88 \%$ of their 192 amino acids with $R A C 3$, respectively (Figure $\mathrm{S} 1$ ). De novo missense variants in RAC1 were recently implicated in causing

\footnotetext{
${ }^{1}$ Division of Clinical and Metabolic Genetics, The Hospital for Sick Children, Toronto, ON, Canada; ${ }^{2}$ Center for Medical Genetics, Ghent University Hospital, Ghent, Belgium; ${ }^{3}$ Center for Genomics and Transcriptomics, Eberhard Karls University of Tübingen, Tübingen, Germany; ${ }^{4}$ Victorian Clinical Genetics Services, Murdoch Children's Research Institute, \& Department of Paediatrics, University of Melbourne, Melbourne, Australia; ${ }^{5}$ The Centre for Applied Genomics, The Hospital for Sick Children, Toronto, ON, Canada; ${ }^{6}$ Neurodevelopmental Genomics Research Group, Murdoch Children's Research Institute, Melbourne, Australia; ${ }^{7}$ VIB-VUB Structural Biology Research Center, Brussels, Belgium; ${ }^{8}$ Department of Paediatrics, University of Toronto, Toronto, ON, Canada; ${ }^{9}$ Division of Paediatric Medicine, The Hospital for Sick Children, Toronto, ON, Canada; ${ }^{10}$ Child Health Evaluative Sciences, The Hospital for Sick Children, Toronto, ON, Canada; ${ }^{11}$ Centre for Genetic Medicine, The Hospital for Sick Children, Toronto, ON, Canada; ${ }^{12}$ Department of Pediatrics, Ghent University, Ghent, Belgium; ${ }^{13}$ Program in Genetics and Genome Biology, The Hospital for Sick Children, Toronto, ON, Canada; ${ }^{14}$ Department of Molecular Genetics, University of Toronto, Toronto, ON, Canada; ${ }^{15}$ Department of Diagnostic Imaging, The Hospital for Sick Children, Toronto, ON, Canada; ${ }^{16}$ The Prenatal Diagnosis and Medical Genetics Program, Mount Sinai Hospital, University of Toronto, Toronto, ON, Canada; ${ }^{17}$ Genome Diagnostics, Department of Paediatric Laboratory Medicine, The Hospital for Sick Children, Toronto, ON, Canada; ${ }^{18}$ Laboratory Medicine and Pathobiology, University of Toronto, Toronto, ON, Canada; ${ }^{19} \mathrm{Center}$ for Human Genomics and Precision Medicine, University of Wisconsin School of Medicine and Public Health, Madison, WI, United States. Correspondence: Gregory Costain (gregory.costain@sickkids.ca) or Christian R. Marshall (crm@sickkids.ca) or M. Stephen Meyn (stephen.meyn@wisc.edu)

These authors contributed equally: Gregory Costain, Bert Callewaert, Heinz Gabriel, Tiong Y. Tan
} 
developmental disorders. ${ }^{4}$ Somatic missense variants at conserved residues that activate RAC subfamily proteins have been found in a variety of adult-onset human tumors and promote transformation of human cells in culture. ${ }^{5,6}$ Through genome-wide sequencing and multinational collaboration, we have identified three such missense variants in RAC3 occurring as de novo constitutional events. One variant was recurrent in unrelated families. The five affected individuals share a phenotype of severe intellectual disability and brain malformations.

\section{MATERIALS AND METHODS}

We recruited the index case for a research study involving children with medical complexity suspected of having undiagnosed genetic conditions. Genome sequencing of the proband and his parents (family I) was performed using highquality blood-derived DNA and our established methods ${ }^{7}$ at the Centre for Applied Genomics (http://www.tcag.ca/; Toronto, ON, Canada). The study was approved by the Research Ethics Board at the Hospital for Sick Children. Discovery of a novel de novo missense variant in RAC3 (GenBank NM_005052.2) in family I prompted a search for similar genetic variations in public and private databases. Four other individuals with de novo missense variants in RAC3 were subsequently identified: family II through MyGene2 (http://mygene2.org/) mediated by MatchMaker Exchange (http://www.matchmakerexchange.org/), family III through ClinVar (http://www.ncbi.nlm.nih.gov/clinvar/), and family IV through GeneMatcher (http://www.genematcher. org/). The proband in family II underwent exome sequencing on DNA extracted from peripheral blood lymphocytes. The RAC3 variant was not identified on subsequent targeted testing of parental DNA samples; both maternity and paternity were confirmed. The proband in family III and his parents underwent exome sequencing on DNA extracted from peripheral blood lymphocytes. The affected individuals in family IV were recruited to the Murdoch Children's Research Institute Undiagnosed Diseases Program for research-based exome sequencing. Because these two children share the same mother but were confirmed to have different paternity, exome sequencing was undertaken using DNA extracted from peripheral blood lymphocytes for both children and their mother. All five affected individuals had normal chromosomal microarray results, and no alternative diagnoses were apparent in their genome-wide sequencing data. Phenotype data derived from direct assessment by study coauthors were supplemented by review of lifetime medical records. Brain magnetic resonance images (MRIs) for four of the five were independently reviewed by a neuroradiologist. Informed consent was obtained for each participant included in this report, including for publication of photographs where applicable.

Each $R A C 3$ variant was queried in the published human and model organism literature, and in large databases of somatic variation such as Catalogue of Somatic Mutations in Cancer (COSMIC v84; http://cancer.sanger.ac.uk/cosmic).
Variation at these conserved residues was also investigated in other members of the Rho GTPase gene family with high sequence homology to RAC3, where the equivalent variant in the different genes results in the same amino acid change. Observations concerning the potential impact of the RAC3 variants on protein conformation were made using $3 \mathrm{D}$ structures from the Protein Data Bank (PDB; http://www. $\mathrm{rcsb}$.org/pdb/). In the 2QME PDB entry, the RAC3 protein binds to a segment of the human p21-activated kinase 1 (PAK1) and to phosphomethylphosphonic acid-guanylate ester (GCP). The 2IC5 PDB entry includes two copies of RAC3, with one (chain A) binding phosphoaminophosphonic acid-guanylate ester (GNP) and the other (chain B) binding GDP.

\section{RESULTS}

Genotype and phenotype details are provided in Table 1. The three different $R A C 3$ variants affect conserved nucleotides and amino acids, are predicted to be damaging by in silico programs, and were absent in all members of the RAC subfamily in gnomAD (Table S1). Family histories were noncontributory and consistent with a de novo inheritance pattern in three instances (Fig. 1). Maternal gonadal mosaicism is suspected in family IV (Fig. 1), because the $R A C 3$ variant was absent from 167 sequencing reads in the phenotypically unaffected mother.

All five individuals with RAC3 variants had structural anomalies on brain MRI. Imaging of the index case identified cerebral dysgenesis, including callosal absence, medial bifrontal polymicrogyria, and unilateral ventriculomegaly with abnormal overlying cortex (Fig. 1). The proband in family II similarly had midline, albeit different, abnormalities (thinned corpus callosum, fused forniceal columns, and septal leaflet deficiency) (Fig. 1), and possible occipital polymicrogyria (not shown). Brain MRI at age 2 years for the proband in family III reportedly showed perisylvian polymicrogyria, hypoplasia of the corpus callosum, and broad lateral ventricles, with the images not available for independent review. The two half-siblings in family IV had Chiari type I malformations, dysgenetic corpora callosa, and single subependymal heterotopic nodules (Fig. 1). The female halfsibling in family IV also had severe hydrocephalus diagnosed in infancy, requiring ventriculoperitoneal shunting. The mother in family IV had brain MRI that demonstrated only mild ventricular asymmetry and no Chiari malformation (data not shown).

Other shared neurological features common to all five participants were global developmental delay, severe to profound intellectual disability, and abnormal tone (Table 1). Four of the subjects currently have a head circumference in the normal range. In family I, the proband had a normal head circumference in early childhood but now has mild microcephaly in adolescence $(52 \mathrm{~cm}$ at 16 years; -2.5 standard deviations). The extraneurologic phenotypes were minimal (Table 1). There were variable nonspecific dysmorphic features, including recurrent features like arched and/or 
Table 1 Genotype and phenotype features of five individuals with novel missense variants in $R A C 3^{a}$

\begin{tabular}{|c|c|c|c|c|c|}
\hline & \multirow{2}{*}{$\begin{array}{l}\text { Family I } \\
\text { Individual } 1\end{array}$} & \multirow{2}{*}{$\begin{array}{l}\text { Family II } \\
\text { Individual } 1^{\mathrm{b}}\end{array}$} & \multirow{2}{*}{$\begin{array}{l}\text { Family III } \\
\text { Individual } 1^{\mathrm{c}}\end{array}$} & \multicolumn{2}{|l|}{ Family IV } \\
\hline & & & & Individual 1 & Individual 2 \\
\hline \multicolumn{6}{|l|}{ Demographics } \\
\hline Age at last assessment & 17 years & 5 years & 5 years & 19 years & 14 years \\
\hline Ethnicity & $\begin{array}{l}\text { European } \\
\text { (Caucasian) }\end{array}$ & $\begin{array}{l}\text { European } \\
\text { (Caucasian) }\end{array}$ & $\begin{array}{l}\text { European } \\
\text { (Caucasian) }\end{array}$ & $\begin{array}{l}\text { European } \\
\text { (Caucasian) }\end{array}$ & $\begin{array}{l}\text { European } \\
\text { (Caucasian) }\end{array}$ \\
\hline Genomic position (Chr17; GRCh37) & g.79990661A>T & g.79990313C>T & g.79990663G>A & g.79990663G $>A$ & g.79990663G>A \\
\hline $\begin{array}{l}\text { Coding DNA position } \\
\text { (NM_005052.2) }\end{array}$ & c. $182 \mathrm{~A}>\mathrm{T}$ & c. $86 C>T$ & c. $184 \mathrm{G}>\mathrm{A}$ & c. $184 \mathrm{G}>\mathrm{A}$ & c. $184 \mathrm{G}>\mathrm{A}$ \\
\hline Protein level change (NP_005043.1) & p.(Gln61Leu) & p.(Pro29Leu) & p.(Glu62Lys) & p.(Glu62Lys) & p.(Glu62Lys) \\
\hline Inheritance & De novo & De novo & De novo & De novo ${ }^{d}$ & De novo ${ }^{d}$ \\
\hline Structural abnormality on brain $\mathrm{MRI}^{\mathrm{e}}$ & Yes & Yes & Yes & Yes & Yes \\
\hline Macro/microcephaly in childhood & No & No & No & No & No \\
\hline Seizures & Yes & Yes & No & No & No \\
\hline \multicolumn{6}{|l|}{ Extraneurologic phenotype } \\
\hline Feeding difficulties & Yes & Yes & No & Yes & Yes \\
\hline Other congenital anomalies ${ }^{f}$ & Yes & No & No & Yes & Yes \\
\hline Malignancy & No & No & No & No & No \\
\hline
\end{tabular}

MRI magnetic resonance image.

${ }^{a}$ Additional phenotype details are available upon request.

${ }^{b}$ Family ID 773 in MyGene2 (http://www.mygene2.org; accessed April 2018).

'Submission accession SCV000575128.1 in ClinVar.

dSuspected maternal gonadal mosaicism.

esee text and Fig. 1 for details.

${ }^{f}$ Family I, individual 1: bilateral inguinal cryptorchidism; family IV, individual 1: unilateral lambdoid craniosynostosis; severe scoliosis (Figure S4); family IV, individual 2: hydrocephalus requiring ventriculoperitoneal shunting; severe scoliosis (Figure S5).

medially flared eyebrows (Fig. 1; Figures S2-S5). At the time of last assessment, none had a personal history of malignancy, but the oldest is only 19 years of age.

In silico protein modeling was used to explore the potential functional impact of the p.(Gln61Leu), p.(Glu62Lys), and p. (Pro29Leu) changes. The residues p.Gln61 and p.Glu62 localize within the Switch II region of RAC3 (Figure S6), which in other Rho GTPases presents a 3(10)-helix that disappears upon activation. ${ }^{2}$ In one of the PDB structures, $\mathrm{p}$. Gln61 makes an H-bond with the phosphate group of GNP (Figure S7). The variation p.(Gln61Leu) will disrupt this $\mathrm{H}$ bond and is also likely to destabilize the small 3(10)-helix at residues 62-64, based on energetic considerations. The $\mathrm{p}$. (Glu62Lys) may similarly lead to a conformational change in this 3(10)-helix, as switching the charge of the sidechain from negative to positive is expected to lead to repulsion from the nearby positively charged p.Lys96 (Figure S8). The p.Pro29 residue is positioned in the Switch I region and may play an important role in limiting its flexibility by helping to "tether" the bound GTP (Figure S9). The alteration p.(Pro29Leu) is expected to induce a conformational change owing to the introduction of the large hydrophobic sidechain and/or to increase the flexibility of the loop, similar to the well-studied p.(Pro29Ser) in RAC1 (ref. ${ }^{5,6}$ ).

\section{DISCUSSION}

Published in vivo and in situ experimental data support a transforming effect for each of the identified variants, particularly the two nonrecurrent variants. The alteration $\mathrm{p}$. (Gln61Leu) has been extensively studied in the RAC subfamily, ${ }^{4,8}$ including in RAC3 (ref. ${ }^{10,11}$ ), as an archetype for constitutive protein activation. At the adjacent amino acid, there is variation reported in somatic mutation databases ${ }^{6}$ and a single report of a constitutional p.(Glu62Lys) change in RAC2 that resulted in a gain of function. ${ }^{12}$ The p.Glu62 residue is known to be a crucial component of Ras, ${ }^{13}$ with additional supportive evidence that p.(Glu62Lys) is a recurrent pathogenic gain-of-function variant in KRAS. ${ }^{14,15}$ Furthermore, the amino acids surrounding p.Glu62 demonstrate local constraint for missense variants. Variation at the p.Pro29 residue in $R A C 1$ is an established driver of melanoma and other malignancies. ${ }^{5,6}$ The specific nucleotide substitution 

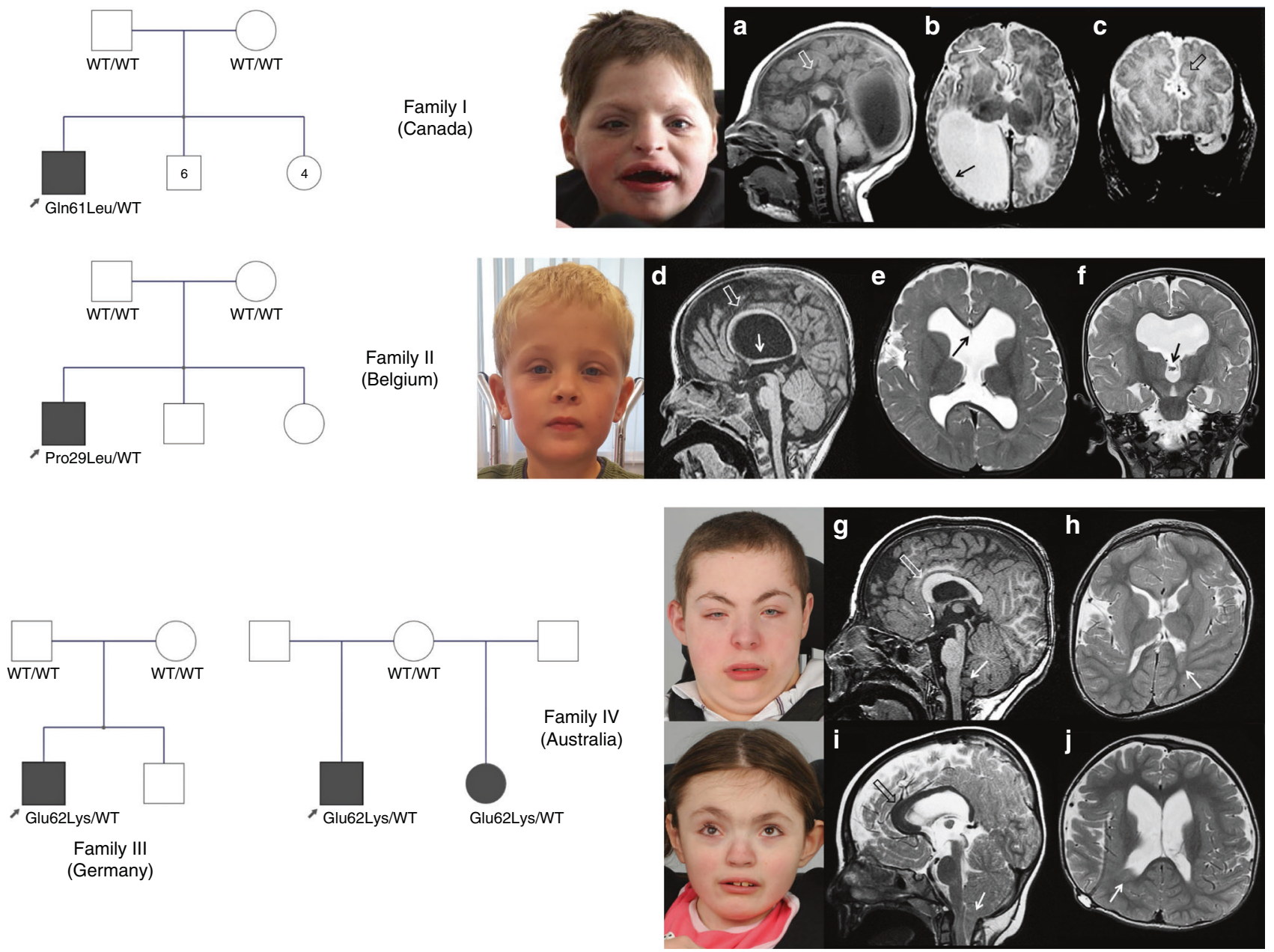

Fig. 1 Five individuals from four families with missense variants in RAC3, severe developmental disorders, and brain malformations. See text and Table 1 for details. Additional photographs of the affected individuals in families I, II, and IV are provided in the Supplemental file (Figures S2-S5). Photographs and brain magnetic resonance images (MRIs) for the proband in family III were not available for publication. He was described as having brachycephaly, hypertelorism, a broad philtrum, and ulnar deviation of wrist joints. In the proband in family I, (a) sagittal T1 weighted image at 4 days of age demonstrates lack of corpus callosum (open arrow), Probst bundles, and cingulate gyrus. (b) Unilateral marked ventriculomegaly of right posterior horn, trigone, and temporal horn are present on T2 weighted axial image. There is marked thinning of the right occipital cortex (black arrow) and medial bifrontal polymicrogyria (white arrow). (c) Coronal T2 weighted image confirms lack of corpus callosum (open arrow). In the proband in family II, (d) sagittal T1 weighted image demonstrates thinning of the corpus callosum (open arrow) and caudal displacement of the forniceal columns (arrow). (e) Axial T2 weighted image shows moderate ventriculomegaly with dehiscent septal leaflets. Only a septal remnant (black arrow) remains. (f) Coronal T2 weighted image again shows ventriculomegaly and incomplete hippocampal rotation. There is fusion of the forniceal columns in the midline (black arrow). For the affected individuals in family IV, (g) sagittal T1 weighted image and (i) T2 weighted image demonstrate a dysgenetic corpus callosum with thick genu (open arrows), absent isthmus, and tapered splenium. Additionally, there is a Chiari 1 malformation (arrow) and ballooning of the aqueduct of Sylvius in each patient. (h) Axial T2 weighted image reveals pointed anterior horns, a heterotopic nodule indenting the left posterior horn (arrow), and posterior positional plagiocephaly. (j) Axial T2 weighted image demonstrates ventriculomegaly with Sylvian fissure shunt, a heterotopic nodule indenting the right posterior horn (arrow), and posterior positional plagiocephaly.

c.86C $>$ T leading to p.(Pro29Leu) was shown in the C. elegans ortholog of RAC3 to result in aberrant axon guidance via an apparent gain-of-function mechanism. ${ }^{16}$

Direct or indirect dysregulation of the best studied Rho GTPases, encoded by RAC1, RHOA, and CDC42, results in aberrant neurodevelopment in a dominant manner (Table S2) (refs. ${ }^{1,4,17}$ ). Differences in the terminal portion of $R A C 3$ are sufficient to confer functional specificity compared with the otherwise highly homologous RAC1 (Figure S1) (ref. ${ }^{18}$ ). However, experimental work has established that RAC3 is also involved in neurogenesis, neuronal migration, and other aspects of cortical development. ${ }^{3}$ For example, expression of a constitutively active form of the chicken ortholog of RAC3 in cultured primary neurons had a major negative effect on neuritogenesis. ${ }^{18}$ Our findings represent the first evidence that missense variants in $R A C 3$ can cause a severe neurodevelopmental phenotype in humans. Converging lines of evidence suggest each of the identified variants may result in constitutive protein activation. Consistent with this proposed mechanism, as opposed to haploinsufficiency, are the further observations that (1) $\mathrm{Rac}^{-1-}$ mice do not show a severe phenotype, ${ }^{19}$ and (2) RAC3 is constrained against missense 
variants $(z=1.97)$ but not loss-of-function variants (probability of loss-of-function intolerance $[\mathrm{pLI}]=0.04$ ) in the ExAC database (http://exac.broadinstitute.org/; accessed April 2018). The molecular mechanisms by which transforming variants in RAC3 may disrupt embryonic neural development are now deserving of further study.

A major challenge posed by genome-wide sequencing in clinical diagnostics is how to prove the causality of a novel de novo variant in a plausible candidate disease gene, found in a single patient, in an efficient and cost-effective manner. Direct variant-specific functional experiments for putative transforming missense changes are beyond the scope of most clinical laboratories. We therefore applied a different approach and rapidly identified additional patients and validated likely causality by in silico modeling and database browsing. Our approach illustrates the growing value of aggregation databases such as MatchMaker Exchange and ClinVar. The sequence homology of RAC3 with RAC1 and RAC2, and with members of the Ras superfamily of small GTPases more generally, allowed for reasonable inferences about the pathogenicity of the three $R A C 3$ variants observed in this study. Our approach of leveraging from pre-existing work in homologs may be applicable in other contexts and facilitate variant interpretation. Also, all three variants appear in databases of genetic somatic variation in cancer. We propose that recurrence in such databases, coupled with absence in large populationbased databases of constitutional variation, is suggestive of a functional effect in the appropriate context.

The first report of de novo missense variants in $R A C 1$ was published in 2017 (ref. $^{4}$ ). One variant was shown to result in constitutive activation: transfecting cells with p.(Tyr64Asp) resulted in significantly increased circularity index and a greater proportion of cells exhibiting lamellipodia or ruffles, similar to the effects seen with p.(Gln61Leu). ${ }^{4,8}$ The seven patients displayed marked clinical heterogeneity in their neurological phenotypes. ${ }^{4}$ The study participant with the $\mathrm{p}$. (Tyr64Asp) change was the only individual with normocephaly and the only individual with polymicrogyria, and thereby showed the most similarities to those in our study. In RAC1 and in RAC3, p.(Tyr64Asp) would be expected to destabilize the same small 3(10)-helix as p.(Gln61Leu) and p. (Glu62Lys).

One individual with a de novo RAC3 missense variant (c.176C>G: p.[Ala59Gly]) was reported in a cohort with Robinow syndrome-like features. ${ }^{20}$ She was described as having some dysmorphic facial features reminiscent of Robinow syndrome, in addition to developmental delay, seizures, and an abnormal electroencephalogram (EEG), and a thin corpus callosum. Although no functional data were presented, the authors postulated that disruption of the Wnt signaling system was the underlying mechanism. This RAC3 variant affects a residue where variants have demonstrated transforming potential in homologous genes. We question whether this individual may be more clinically similar to our cohort with a distinct RAC3-related neurodevelopmental disorder than to those with Robinow syndrome.
Taken together, our data suggest that missense variants in RAC3 cause severe intellectual disability and brain malformations. Our experience highlights the importance of basic science discoveries in rapidly translating novel genomic findings into clinical practice, and provides further impetus for the continued study of the role of RAC3 in the central nervous system.

\section{ELECTRONIC SUPPLEMENTARY MATERIAL}

The online version of this article (https://doi.org/10.1038/s41436018-0323-y) contains supplementary material, which is available to authorized users.

\section{ACKNOWLEDGEMENTS}

The research conducted at The Hospital for Sick Children was funded by the Norm Saunders Complex Care Initiative, the Centre for Genetic Medicine, the Centre for Applied Genomics, the Hospital for Sick Children, Genome Canada, and the University of Toronto McLaughlin Centre. B.C. is a senior clinical investigator of the Research Foundation-Flanders. Funding for the UDP-Vic was provided by philanthropic donation and the Murdoch Children's Research Institute. The research conducted at the Murdoch Children's Research Institute was supported by the Victorian Government's Operational Infrastructure Support Program. The funding bodies played no role in the design of the study, the collection, analysis, and interpretation of data, or the writing of the manuscript.

\section{DISCLOSURE}

The authors declare no conflicts of interest.

\section{REFERENCES}

1. Stankiewicz TR, Linseman DA. Rho family GTPases: key players in neuronal development, neuronal survival, and neurodegeneration. Front Cell Neurosci. 2014;8:314.

2. Vetter IR, Wittinghofer A. The guanine nucleotide-binding switch in three dimensions. Science. 2001;294:1299-1304.

3. de Curtis I. Roles of Rac1 and Rac3 GTPases during the development of cortical and hippocampal GABAergic interneurons. Front Cell Neurosci. 2014;8:307.

4. Reijnders MRF, Ansor NM, Kousi M, et al. RAC1 Missense Mutations in Developmental Disorders with Diverse Phenotypes. Am J Hum Genet. 2017; 101:466-477

5. Kawazu M, Ueno T, Kontani K, et al. Transforming mutations of RAC guanosine triphosphatases in human cancers. Proc Natl Acad Sci USA. 2013;110:3029-3034.

6. Porter AP, Papaioannou A, Malliri A. Deregulation of Rho GTPases in cancer. Small GTPases. 2016;7:123-138.

7. Lionel AC, Costain G, Monfared N, et al. Improved diagnostic yield compared with targeted gene sequencing panels suggests a role for whole-genome sequencing as a first-tier genetic test. Genet Med. 2018;20:435-443

8. Michiels F, Habets GG, Stam JC, et al. A role for Rac in Tiam1-induced membrane ruffling and invasion. Nature. 1995;375:338-340.

9. Machesky LM, Hall A. Role of actin polymerization and adhesion to extracellular matrix in Rac- and Rho-induced cytoskeletal reorganization. J Cell Biol. 1997;138:913-926.

10. Joyce $\mathrm{PL}$, Cox $A D$. Rac1 and Rac3 are targets for geranylgeranyltransferase I inhibitor-mediated inhibition of signaling, transformation, and membrane ruffling. Cancer Res. 2003;63:7959-7967.

11. Rosenberg BJ, Gil-Henn H, Mader CC, et al. Phosphorylated cortactin recruits Vav2 guanine nucleotide exchange factor to activate Rac3 and 
promote invadopodial function in invasive breast cancer cells. Mol Biol Cell. 2017;28:1347-1360.

12. Hsu AP Novel heterozygous RAC2 mutation presenting as combined immunodeficiency. Abstract presented at Clinical Immunology Society 2016 Annual Meeting. April 14-17, 2016: Boston, Massachusetts, USA

13. Gasper R, Thomas C, Ahmadian MR, et al. The role of the conserved switch II glutamate in guanine nucleotide exchange factor-mediated nucleotide exchange of GTP-binding proteins. J Mol Biol. 2008;379:51-63.

14. Niu $B$, Scott AD, Sengupta $S$, et al. Protein-structure-guided discovery of functional mutations across 19 cancer types. Nat Genet. 2016;48:827-837.

15. Kim E, llic N, Shrestha $Y$, et al. Systematic functional interrogation of rare cancer variants identifies oncogenic alleles. Cancer Discov. 2016;6:714-726.

16. Shakir MA, Gill JS, Lundquist EA. Interactions of UNC-34 enabled with Rac GTPases and the NIK kinase MIG-15 in Caenorhabditis elegans axon pathfinding and neuronal migration. Genetics. 2006;172:893-913.
17. Martinelli S, Krumbach OHF, Pantaleoni $F$, et al. Functional dysregulation of CDC42 causes diverse developmental phenotypes. Am J Hum Genet 2018; pii: S0002-9297(17)30504-9. https://doi.org/10.1016/j.ajhg. 2017.12.015. [Epub ahead of print].

18. Albertinazzi C, Gilardelli D, Paris $S$, et al. Overexpression of a neuralspecific rho family GTPase, CRac1B, selectively induces enhanced neuritogenesis and neurite branching in primary neurons. J Cell Biol. 1998; 142:815-825.

19. Pennucci R, Talpo F, Astro $V$, et al. Loss of either Rac1 or Rac3 GTPase differentially affects the behavior of mutant mice and the development of functional GABAergic networks. Cereb Cortex. 2016;26: $873-890$

20. White JJ, Mazzeu JF, Coban-Akdemir Z, et al. WNT signaling perturbations underlie the genetic heterogeneity of Robinow syndrome. Am J Hum Genet. 2018;102:27-43. 